\title{
DÜBLIN
}

Technological University Dublin

ARROW@TU Dublin

\section{On the Evolution Equation for Modelling the Covid-19 Pandemic}

Jonathan Blackledge

Technological University Dublin, jonathan.blackledge@tudublin.ie

Follow this and additional works at: https://arrow.tudublin.ie/engschelebk

Part of the Analysis Commons, Applied Statistics Commons, Probability Commons, and the Statistical Models Commons

\section{Recommended Citation}

Blackledge J.M. (2021) On the Evolution Equation for Modelling the Covid-19 Pandemic. In: Agarwal P., Nieto J.J., Ruzhansky M., Torres D.F.M. (eds) Analysis of Infectious Disease Problems (Covid-19) and Their Global Impact. Infosys Science Foundation Series. Springer, Singapore. https://doi.org/10.1007/ 978-981-16-2450-6_4

This Book Chapter is brought to you for free and open access by the School of Electrical and Electronic Engineering at ARROW@TU Dublin. It has been accepted for inclusion in Books/Book chapters by an authorized administrator of ARROW@TU Dublin. For more information, please contact arrow.admin@tudublin.ie, aisling.coyne@tudublin.ie, gerard.connolly@tudublin.ie.

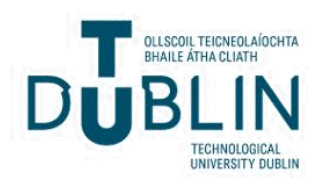




\title{
On the Evolution Equation for Modelling the Covid-19 Pandemic
}

\author{
J. M. Blackledge
}

\begin{abstract}
The paper introduces and discusses the evolution equation, and, based exclusively on this equation, considers random walk models for the time series available on the daily confirmed Covid-19 cases for different countries. It is shown that a conventional random walk model is not consistent with the current global pandemic time series data, which exhibits non-ergodic properties. A self-affine random walk field model is investigated, derived from the evolutionary equation for a specified memory function which provides the non-ergodic fields evident in the available Covid-19 data. This is based on using a spectral scaling relationship of the type $1 / \omega^{\alpha}$ where $\omega$ is the angular frequency and $\alpha \in(0,1)$ conforms to the absolute values of a normalised zero mean Gaussian distribution. It is shown that $\alpha$ is a primary parameter for evaluating the global status of the pandemic in the sense that the pandemic will become extinguished as $\alpha \rightarrow 0$ for all countries. For this reason, and based on the data currently available, a study is made of the variations in $\alpha$ for 100 randomly selected countries. Finally, in the context of the Bio-dynamic Hypothesis, a parametric model is considered for simulating the three-dimensional structure of a spike protein which may be of value in the development of a vaccine.
\end{abstract}

Keywords Einstein's Evolution equation - Self-Affine random walk fields • Pandemic time series analysis $\cdot$ Bio-dynamics hypothesis $\cdot$ Fractal geometry of spike proteins.

\footnotetext{
J. M. Blackledge ( $\square)$

School of Mathematics, Statistics and Computer Science, University of KwaZulu-Natal,

KwaZulu-Natal, South Africa

e-mail: jonathan.blackledge@TUDublin.ie
}

School of Electrical and Electronic Engineering, Technological University Dublin, Dublin, Ireland Department of Computer Science, University of Western Cape, Western Cape, South Africa

Faculty of Arts, Science and Technology, Wrexham Glyndrw University of Wales, Wales, UK

Centre for Advanced Studies, Warsaw University of Technology, Warsaw, Poland

Science Foundation Ireland, Dublin, Ireland

(C) The Author(s), under exclusive license to Springer Nature Singapore Pte Ltd. 2021

P. Agarwal et al. (eds.), Analysis of Infectious Disease Problems (Covid-19)

and Their Global Impact, Infosys Science Foundation Series,

https://doi.org/10.1007/978-981-16-2450-6_4 


\section{Introduction}

Coronaviruses are a family of viruses that can cause illnesses from the common cold to severe acute respiratory syndrome. In late 2019, a new coronavirus was identified as the cause of a disease outbreak that originated in China. The virus is now known as the severe acute respiratory syndrome coronavirus 2 (SARS-CoV-2). The disease it causes is called coronavirus disease 2019 (Covid-19), and, in March 2020, the World Health Organisation declared the Covid-19 outbreak a pandemic [1]. A pandemic is an epidemic of an infectious disease that spreads across large regions such as multiple continents or worldwide, affecting a substantial number of people. They are a natural occurrence of human evolution, made more probable by the increasing size of the human population and its propensity to localisation in urban centres. Past pandemics were caused by diseases such as Small Pox and Tuberculosis. The most fatal pandemic in recorded history was the Black Death (The Plague), which killed an estimated 75-200 million people in the $14^{\text {th }}$ Century. The most notable pandemic of the last century occurred over 100 years ago, namely the influenza pandemic (Spanish flu) which killed an estimated 50-100 million people worldwide.

The 1918 Spanish flu pandemic initiated some of the earliest work on the mathematical modelling of infectious diseases. Since the pioneering work of Kermack and McKendrick in 1926-27 [2], modelling the dynamics of disease transmission has been based on the development of increasingly complex systems of differential equations. A fundamental property of such models is the Basic Reproduction Number which is a threshold value, below which an infectious disease cannot spread in a susceptible population. This number needs to be less than one for an infectious disease to be extinguished.

In the context of any infectious disease, and, an associated vaccination program (if one exists), the concept of herd immunity is fundamental [3]. This is because with herd immunity, it is not necessary to vaccinate an entire population in order to reduce the progress and possibly eliminate an infectious disease, a concept that proved its value during the eradication of Smallpox in the 1970 s, for example. Since then, mathematical modelling has become increasingly important for public health policy making. This has included the control of the human immunodeficiency viruses in terms of predicting the further course of the epidemic and trying to identify the most effective prevention strategies. With further infectious disease outbreaks such as the Severe Acute Respiratory Syndrome virus of 2002, Swine Flu in 2009, and, more recently, the outbreak of Covid-19, infectious disease models have become increasingly sophisticated, e.g. [4, 5].

Mathematical models that are based on systems of differential equation are examples of deterministic models where each term in the equation plays a part in modelling a specific component of a dynamical process. Such models depend on multiple parameters (coefficients and initial conditions, for example) that affect the solutions obtained. This is because each term that is included is usually predicated on some coefficient which needs to be known relatively accurately and may vary in time. In the absence of accurate values for these coefficients, some of which may be known 
unknown's, these models can output error prone results in terms of predicted outcomes as a function of time. In the current Covid-19 pandemic, the sophistication of such models has been extended further to incorporate more and more effects in line with the policies and practices implemented by central government and local health authorities. The sophistication of such models increases further the parameter sets whose values require increasingly accurate estimates. This problem is typical of situations in mathematical modelling where deterministic models become too complex to provide future forecasts that are accurate enough to be of significance [6]. Further, if these models include nonlinear terms, it is likely that the output(s) may be become chaotic. In this case, the determinism of such models becomes an irrelevance and we must turn to the application of stochastic modelling methods which is the subject of this work.

In this paper, an approach is explored whose aim it to model a set of time series (Covid-19 daily cases, for example) but not in terms of predicting a specific outcome for a specific country at a point in time. Instead we focus on modelling the global random walk field for the current Covid-19 pandemic. This is where the assembly of all time series for all countries is treated as a set of random walks to produce a 'random walk field'. The aim is to simulate this field in a way that is representative of the known data and to show that it is analogous to the interaction of a canonical ensemble of particles each undergoing random motion over a period of time. In this context, it is assumed that the pandemic will eventually diffuse throughout the world population and become a steady state effect rather than be fully eradicated, and, that any intervention in this process will only delay the final steady state condition rather than extinguish it.

A random walk model is developed which illustrates that the Covid-19 data fields available (i.e. the set of time series data on daily cases) are not representative of classical diffusion [7] but of fractional diffusion [8]. The development of this model is predicated on an analysis of the evolution equation as are all the results presented in the paper. This allows the models developed to be understood in the context of a fundamental field equation of statistical physics as discussed in the following section. We then use this field equation to develop models for ergodic random and non-ergodic self-affine random walk fields which is the subject of Sect. 3 and Sect. 4, respectively. This is followed by the introduction of a parametric solution for modelling the structural complexity of the viral spike protein which may have ramifications in the development of a vaccine subject to the Bio-dynamics Hypothesis as presented in Sect. 5.

The Bio-dynamics Hypothesis is the result of asking a simple question: why are so many biological entities, irrespective of their physical scale or origin, composed of self-affine structures? In the context of this question, the hypothesis states that: If replication is a self-affine process of time, then the geometrical structure of the result is also self-affine [9]. The hypothesis attempts to relate the dynamical behaviour of replication, mutation and evolutionary biology to the self-affine structure (the fractal structure) of biological entities. In this paper, we present some results from an analysis of Covid-19-based data assuming that the pandemic is a self-organising 
processes, a result that is derived specifically from the evolution equation. This approach complements the analysis of the pandemic based on self-organising maps, for example [10].

\section{The Evolution Equation}

Let $p(\mathbf{r})$ denote a Probability Density Function (PDF) where

$$
\int_{-\infty}^{\infty} p(\mathbf{r}) d^{n} \mathbf{r}=1
$$

which characterises the position of particles in a $n$-dimensional space $\mathbf{r} \in \mathbb{R}^{n}$ ( $n=$ $1,2,3)$. At any instant in time $t$, the particles are distributed in space as a result of some 'random walk' process involving elastic interactions or 'elastic scattering' (with other like particles in the same $n$-dimensional space, when, in all cases, both momentum and energy are conserved). Let $u(\mathbf{r}, t)$ denote the density function (i.e. the number of particles per unit of an $n$-dimensional space) associated with a canonical assemble of particles all undergoing the same random walk processes.

Consider an initial condition where we have an infinitely small concentration of such particles at a time $t=0$ located at an origin $\mathbf{r}=\mathbf{0}$. The density function at $t=0$ is then given by $u(\mathbf{r}, 0)=\delta^{n}(\mathbf{r})$ where $\delta^{n}(\mathbf{r})$ is the $n$-dimensional Dirac delta function. At some short time later $t<<1$, it can be expected that the density function will be determined by the PDF governing the distribution of particles after a (short duration) random walk. Thus we can write

$$
u(\mathbf{r}, t)=p(\mathbf{r}) \otimes u(\mathbf{r}, 0)=p(\mathbf{r}) \otimes \delta^{n}(\mathbf{r})=p(\mathbf{r})
$$

where $\otimes$ denotes the convolution integral over $\mathbf{r}$. The PDF $p(\mathbf{r})$ therefore represents the response (in a statistical sense) to a short time random walk process, and, in this context, can be taken to be is a distributional Impulse Response Function (IRF). Thus, for any time $t$, the density field at some later time $t+\tau$ will be given by

$$
u(\mathbf{r}, t+\tau)=p(\mathbf{r}) \otimes u(\mathbf{r}, t)
$$

For any instant in time $t$, Eq. (1) shows that the spatial behaviour of the density field at some future time $\tau$ is given by the convolution of the density of particles at a previous time with the PDF of the system that governs its 'statistical evolution'. In this sense, $p(\mathbf{r})$ is analogous to the IRF of a linear stationary system when, for an initial condition $u_{0}(\mathbf{r}) \equiv u(\mathbf{r}, t=0)$, say,

$$
u(\mathbf{r}, t)=g(r, t) \otimes u_{0}(\mathbf{r})
$$


where $g(r, t)$ is the characteristic Green's function of the system. However, in this case $u(\mathbf{r}, t)$ denotes a deterministic function associated with the behaviour of a deterministic system, whereas in Eq. (1), $u(\mathbf{r}, t)$ is the density function associated with the evolution of a distribution for a stochastic system. This 'system' is taken to be stationary in a statistical sense because it is assumed that $p(\mathbf{r})$ does not vary in time and the time evolution model given by Eq. (1) is referred to as being 'Ergodic'. Further, we note that if the PDF is symmetric, then $p(\mathbf{r}) \equiv p(r)$ where $r=|\mathbf{r}|$.

Equation (1) is an evolution equation first derived by Albert Einstein in 1905 [11]. It is the principal field equation for elastic scattering processes in statistical mechanics and is an example of a continuous time random walk model where $p(\mathbf{r})$ is the PDF for the displacement $\mathbf{r}$ of a particles position over time interval $\tau$. For some stochastic source function $s(\mathbf{r}, t)$, the evolution equation is generalised further to the form

$$
u(\mathbf{r}, t+\tau)=p(\mathbf{r}) \otimes u(\mathbf{r}, t)+s(\mathbf{r}, t)
$$

This equation describes the evolution of the density function $u(\mathbf{r}, t)$ when the initial particle concentration is replenished in space and/or time and can be extended further to include a decay factor over time when it is required to consider an evolution equation of the type (for decay rate factor $\lambda$, say)

$$
u(\mathbf{r}, t+\tau)=p(\mathbf{r}) \otimes u(\mathbf{r}, t)+s(\mathbf{r}, t)-\lambda u(\mathbf{r}, t)
$$

In this paper, we focus exclusively on the application of Eq. (2) for modelling and analysing time series data associated with the Covid-19 pandemic. In this application, the density field $u$ is taken to be the number of infections divided by the number of those at risk to infection.

One of the purposes of this paper is to bring to the attention of the reader the value of using Eq. (2) to develop a unified framework for stochastic modelling in public health medicine. In this context, there are two other equations which, although essentially different ways of writing Eq. (2), are nevertheless informative, especially in regard to understanding some of the consequences of imposing certain condition on Eq. (2) and the interpretation of the results that follow. These equations are discussed in the following sections.

\subsection{The Classical Kolmogorov-Feller Equation}

Consider the following Taylor series for the function $u(\mathbf{r}, t+\tau)$ in Eq. (2):

$$
u(\mathbf{r}, t+\tau)=u(\mathbf{r}, t)+\tau \frac{\partial}{\partial t} u(\mathbf{r}, t)+\frac{\tau^{2}}{2 !} \frac{\partial^{2}}{\partial t^{2}} u(\mathbf{r}, t)+\ldots
$$

For $\tau<<1$ 


$$
u(\mathbf{r}, t+\tau) \simeq u(\mathbf{r}, t)+\tau \frac{\partial}{\partial t} u(\mathbf{r}, t)
$$

and from Eq. (2), we obtain the Classical Kolmogorov-Feller Equation (CKFE), [13, $14]$

$$
\tau \frac{\partial}{\partial t} u(\mathbf{r}, t)=-u(\mathbf{r}, t)+u(\mathbf{r}, t) \otimes p(\mathbf{r})+s(\mathbf{r}, t)
$$

which is a representation of Eq. (2) when $\tau<<1$.

Equation (3) is based on a critical assumption which is that the time evolution of the density field $u(\mathbf{r}, t)$ is influenced only by short term events and that longer term events have no influence on the behaviour of the field at any later time. This is to say that the 'system' described by Eq. (3) has no 'memory'. This statement is the physical basis upon which the condition $\tau<<1$ is imposed, thereby facilitating the Taylor series expansion of the function $u(\mathbf{r}, t+\tau)$ to first order alone. It means that if a time series is taken to be described by $u(t)$ (for some fixed position in space) then the behaviour of this time series at any time $t$ is not influenced by its behaviour at some earlier time less that $t$. This is the basis for understanding classical diffusion, for example, when Eq. (3) can be used to derive the classical diffusion equation given that $p(\mathbf{r})$ is a Gaussian distribution. For $\mathbf{r} \in \mathbb{R}^{n}$, this can be shown by approximating the Characteristic Function for a Gaussian distribution (which is itself Gaussian). For example, if we consider the case when the source function is zero and apply the approximation $\exp \left(-|\mathbf{k}|^{2}\right) \simeq 1-k^{2}$, then, in Fourier space, Eq. (3) is given by

$$
\tau \frac{\partial}{\partial t} U(\mathbf{k}, t)=-k^{2} U(\mathbf{k}, t)
$$

which is a Fourier space representation of the classical diffusion equation

$$
\frac{\partial}{\partial t} u(\mathbf{r}, t)=D \nabla^{2} u(\mathbf{r}, t)
$$

where $D=1 / \tau$ is the diffusivity, i.e. a measure of the rate at which particles can spread. The Green's function solution to the diffusion equation is [15]

$$
u(\mathbf{r}, t)=g(r, t) \otimes u_{0}(\mathbf{r})
$$

where $g(r, t)$ is the Green's function given by

$$
g(r, t)=\left(\frac{1}{4 \pi D t}\right)^{\frac{n}{2}} \exp \left(-\frac{r^{2}}{4 D t}\right), t \geq 0
$$

and $u_{0}(\mathbf{r})=u(\mathbf{r}, t=0)$ is the initial condition. On the basis of this solution, we can infer that as $\tau$ increases, the speed of diffusion decreases, i.e. the distribution of $u(\mathbf{r}, t)$ in time is slower for larger values of $\tau$. The equivalent solution to Eq. (3) which is inclusive of the source function is 


$$
u(\mathbf{r}, t)=g(r, t) \otimes u_{0}(\mathbf{r})+\int_{0}^{t} g(\mathbf{r}, t-\tau) \otimes s(\mathbf{r}, t) d \tau
$$

Thus, if we consider an asymptotic solution when $r \rightarrow 0$, then for $u_{0}(\mathbf{r})=0$ we obtain

$$
u(t)=\left(\frac{1}{4 \pi D t}\right)^{\frac{n}{2}} \otimes s(t), s(t) \equiv s(0, t)
$$

where $\otimes$ denotes the casual convolution integral in time. This result is an example of a continuous time random walk model for a stochastic time source $s(t)$ which, for classical diffusion, is characterised by scaling factor $1 / t^{n / 2}$.

\subsection{The Generalised Kolmogorov-Feller Equation}

Given that Eq. (3) is memory invariant, the question arises as to how longer temporal influences can be modelled, other than by taking an increasingly larger number of terms in the Taylor expansion of $u(\mathbf{r}, t+\tau)$ which is not analytically consequential, i.e. writing Eq. (2) in the form

$$
\tau \frac{\partial}{\partial t} u(\mathbf{r}, t)+\frac{\tau^{2}}{2 !} \frac{\partial^{2}}{\partial t^{2}} u(\mathbf{r}, t)+\ldots=-u(\mathbf{r}, t)+u(\mathbf{r}, t) \otimes p(r)+s(\mathbf{r}, t)
$$

The key to solving this problem is to consider the idea of expressing the Taylor series on the left-hand side of the equation above in terms of a 'memory function' $m(t)$ and write

$$
\tau m(t) \otimes \frac{\partial}{\partial t} u(\mathbf{r}, t)=-u(\mathbf{r}, t)+u(\mathbf{r}, t) \otimes p(r)+s(\mathbf{r}, t)
$$

This is the generalised Kolmogorov-Feller equation (GKFE). In addition to specifying the source function and the PDF in order to develop a solution for $u$, this equation also requires a memory function to be specified. In this case, if a time series is taken to be described by $u(t)$ (for a fixed position in space) then the behaviour at a time $t$ is influenced by the behaviour at some earlier time according to the characteristics of the memory function. This is an example of a stochastic process in which the past influences the future. The time scale over which this effect is possible then depends on the 'width' in time of the memory function where it is noted that the GKFE reduces to the CKFE when $m(t)=\delta(t)$ which is equivalent to imposing the condition $\tau<<1$. 


\subsection{Orthonormal Memory Functions}

For any inverse function or class of inverse functions of the type $n(t)$, say, such that

$$
n(t) \otimes m(t)=\delta(t)
$$

the GKFE can be written in the form

$$
\tau \frac{\partial}{\partial t} u(\mathbf{r}, t)=-n(t) \otimes u(\mathbf{r}, t)+n(t) \otimes u(\mathbf{r}, t) \otimes p(\mathbf{r})+n(t) \otimes s(\mathbf{r}, t)
$$

where the CKFE is again recovered when $n(t)=\delta(t)$ given that $\delta(t) \otimes \delta(t)=\delta(t)$. The function $n(t)$ is a orthonormal function of $m(t)$. Writing the GKFE in this form facilitates the development of solutions for $u(\mathbf{r}, t)$ given that $n(t)$ can be derived from $m(t)$. In principle, this is possible, given that in Fourier space, the orthonormality relationship between $m(t)$ and $n(t)$ is (using the convolution theorem)

$$
N(\omega)=\frac{1}{M(\omega)}
$$

where $N(\omega)$ and $M(\omega)$ are the Fourier transforms of $n(t)$ and $m(t)$, respectively.

\subsection{Time Series Models}

Equation (5) is a description for a density field that is dependent on both space and time. Given that we are interested in analysing data that are time series alone, it is necessary to develop a time-only series model. A conditional example of this is to note that when $p(\mathbf{r})=\delta^{n}(\mathbf{r})$, we can write Eq. (5) as

$$
\tau \frac{d}{d t} u(t)=n(t) \otimes s(t)
$$

where

$$
u(t)=\int u(\mathbf{r}, t) d^{n} \mathbf{r} \text { and } s(t)=\int s(\mathbf{r}, t) d^{n} \mathbf{r}
$$

To generalise this result further, we consider an asymptotic result for the spatial component of Eq. (5). To do this, we note that using a Taylor expansion for the convolution integral over $\mathbf{r}$, we can write

$$
u(\mathbf{r}, t) \otimes p(\mathbf{r})=\int p(\mathbf{r}-\mathbf{s}) u(\mathbf{s}, t) d^{n} \mathbf{s}=\int[p(\mathbf{r})-\mathbf{s} \cdot \nabla p(\mathbf{r})+\ldots] u(\mathbf{s}, t) d^{n} \mathbf{s}
$$




$$
=p(\mathbf{r}) \int u(\mathbf{s}, t) d^{n} \mathbf{s}+\nabla p(\mathbf{r}) \cdot\left[\int \mathbf{s} u(\mathbf{s}, t) d^{n} \mathbf{s}\right]+\ldots \sim p(\mathbf{r}) \int u(\mathbf{s}, t) d^{n} \mathbf{s}
$$

If the PDF is such that $p(\mathbf{r}) \rightarrow 0$ as $r \rightarrow \infty$, and, in addition, we can assume that the gradient of $p(\mathbf{r})$ and all higher-order gradients approach zero in the same limit, then, given the above series, we can consider the case where

$$
u(\mathbf{r}, t) \otimes p(\mathbf{r}) \sim 0, r \rightarrow \infty
$$

The contribution of the term $n(t) \otimes u(\mathbf{r}, t) \otimes p(\mathbf{r})$ in Eq. (5) then becomes insignificant, and we can consider the time-only dependent asymptotic equation

$$
\tau \frac{d}{d t} u(t)=-n(t) \otimes u(t)+n(t) \otimes s(t)
$$

where $u(t) \equiv u(\mathbf{r}, t), r \rightarrow \infty$ and $s(t) \equiv s(\mathbf{r}, t), r \rightarrow \infty$.

The essential difference between Eqs. (8) and (7) is compounded in the inclusion or otherwise of the term $-n(t) \otimes u(t)$, respectively. In the latter case, i.e. Eq. (8), the spectral response of $u(t)$ to $s(t)$ is determined by the transfer function

$$
T(\omega)=\frac{N(\omega)}{N(\omega)+i \omega \tau}
$$

In the former case, i.e. Eq. (7), the transfer function is

$$
T(\omega)=\frac{N(\omega)}{i \omega \tau}
$$

In both cases, the stochastic behaviour of the density field $u(t)$ depends on the source function $s(t)$ and the memory function $m(t)$.

\subsection{Logarithmic Scale Analysis}

If we let $u=\log w$, then Eq. (7) becomes

$$
\tau \frac{d}{d t} \log w(t)=n(t) \otimes s(t)
$$

By way of an example, consider case when $n(t)=\delta(t)$ and

$$
\tau \frac{d}{d t} \log w(t)=s(t)
$$


Then, for a constant value of $s(t)= \pm s_{0} \forall t$ say, we obtain the standard exponential growth/decay model when, for some initial condition $w_{0}=w(t=0)$,

$$
w(t)=w_{0} \exp \left( \pm s_{0} t / \tau\right)
$$

Thus, we observe that this most basic of time evolution models (exponential growth/decay) is in fact, just a conditional model of the evolution equation when $p(\mathbf{r})=\delta^{n}(\mathbf{r})$ and $n(t)=\delta(t)$ where the density field is taken to be on a logarithmic scale. For a time varying source function, over some interval of time $t$, the solution is

$$
w(t)=\exp \left[\frac{1}{\tau} \int^{t} s(\xi) d \xi\right]
$$

\section{Random Walk Fields}

Consider the example data given in Fig. 1 which shows the daily new confirmed Covid-19 cases (for approximately 200 days) on a linear scale for different countries [16]. The plots provide the rolling 7-day average where the number of confirmed cases is taken to be lower than the number of actual cases due to limited testing. One of the purposes of applying a rolling average is to eliminate the characteristic and

\section{Daily new confirmed COVID-19 cases}
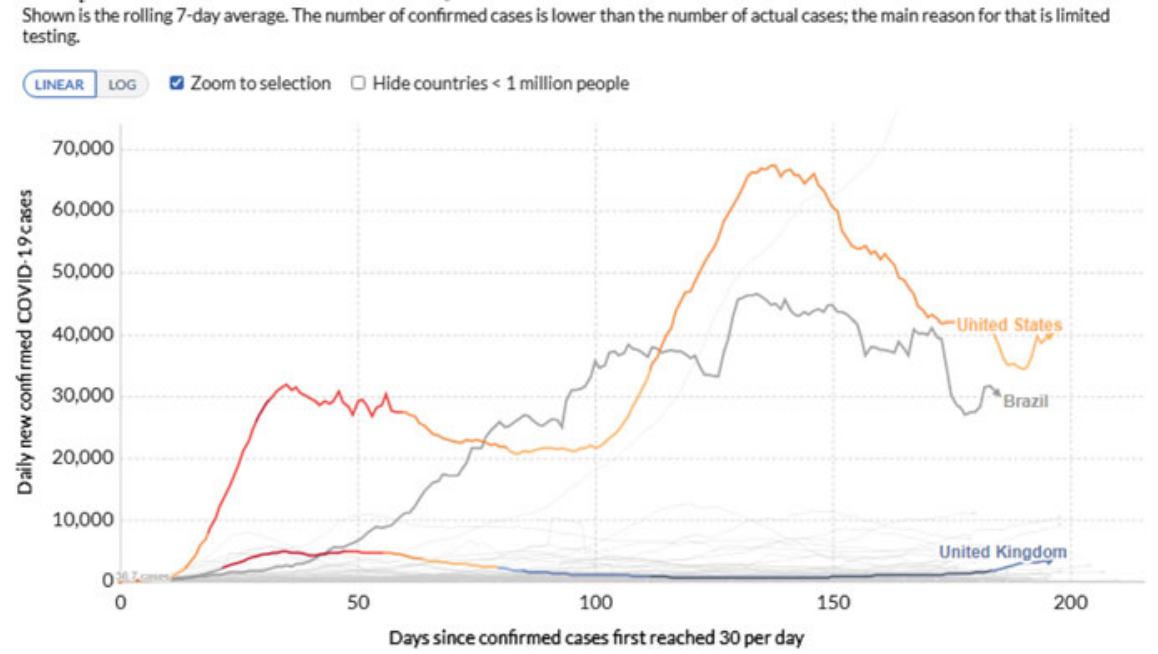

Fig. 1 Daily confirmed Covid-19 cases (for approximately 200 days) on a linear scale for a range of different countries with highlights for the UK, the USA and Brazil [16] 
periodic decrease on the daily rates that occurs over the weekends, at least for the majority of countries when a Saturday and a Sunday are non-working days for the majority of the population. The data must be considered to contain a wide range of errors (e.g. false negatives and false positives), and, on a country by country basis, to have substantial differences in accuracy, consistency and relevance. In this context, Fig. 1 highlights three specific times series for cases in the UK, the USA and Brazil to illustrated some extreme example differences in the progression of the disease for three different countries.

The reasons for the differences in the growth (and decay) of the disease on a country-by-country basis are multifaceted. They are due to differences in the health systems of each country, their social-economic characteristics, the genetic dispositions of the population, age range and immunity signatures, etc. as well as the production and management of the data and the different policies adopted by central governments to control the disease. Thus, the data provided in Fig. 1 cannot be assumed to be an fully accurate representation for any case or to have a uniformity in its inaccuracies across the range of countries given. In this regard, the purpose of this section is to show that in a global context, the behaviour of the pandemic from one country to another appears to reflect a random walk process (a stochastic time series). Taking all the times series given in Fig. 1 produces a random walk field. It is this field that is a focus of the mathematical modelling considered in this work.

In terms of Eq. (7), the random walk model that is now considered is predicated on a one-dimensional model when $\tau<<1$, i.e. the system has no memory so that $n(t)=$ $\delta(t)$. It therefore represents a model in which the simulation of the results given in Fig. 1 are based on the assumption that any interventions imposed or otherwise by a central authority are irrelevant and that the evolution of the pandemic is independent of any other factors such a asymmetry, herd immunity and the effects of a vaccination program, for example. In this case, Eq. (7) reduces to the simplest of evolution equations, namely,

$$
\tau \frac{d}{d t} u(t)=s(t)
$$

Fig. 2 provides an example of a typical set of random walks - a random walk field consisting of 15 trajectories over 200 steps re-scaled to 1 . This results are based on using Euler's method and forward differencing the gradient in time for Eq. (9) to produce the difference equation

$$
u_{n+1}=u_{n}+\Delta s_{n}, n=1,2, \ldots, N-1
$$

with $\Delta=0.001$. Each trajectory follows a different random path from a common initial condition $u_{1}=0$. In this case, $s_{n}$ is taken to be a Gaussian distributed (discrete) variable with a mean of zero. The random walks therefore have both positive and negative amplitudes.

The random walk field illustrated in Fig. 2 spreads out over time, the difference from one trajectory to the next being due to different initial conditions used to seed the Gaussian random number generator (in this example, the MATLAB function 

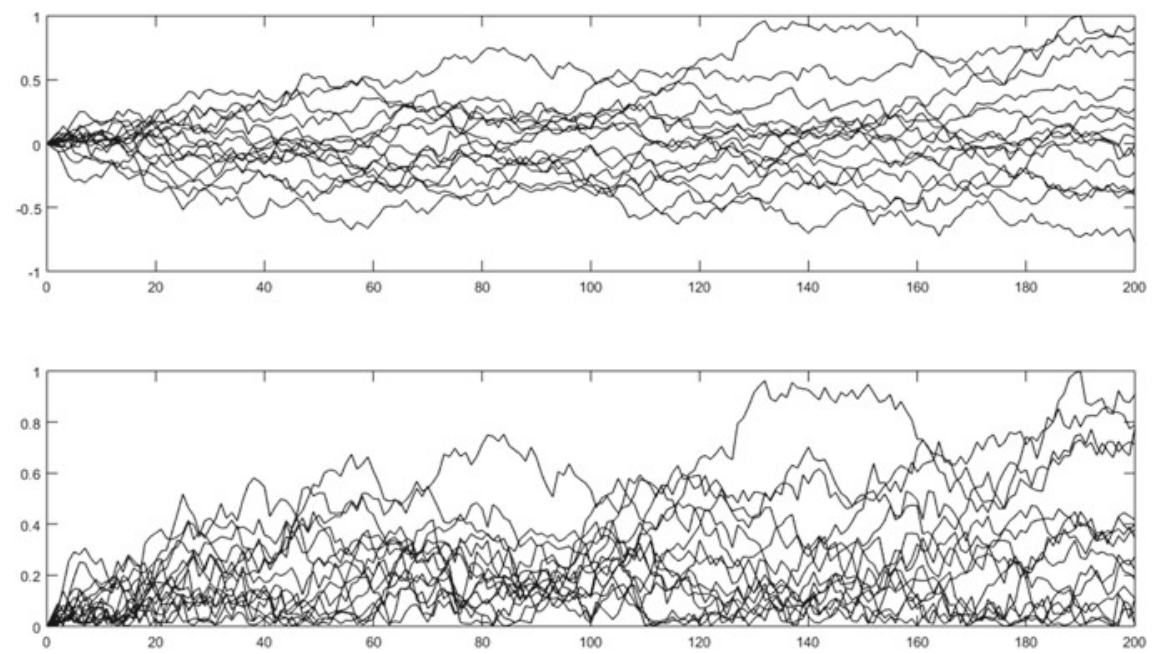

Fig. 2 Simulation of a random walk field illustrating random walks for $u_{n}$ (above) and $\left|u_{n}\right|$ based on Eq. (9) for a random Gaussian distributed source $s_{n}$

randn has been applied). The result is to produce a random walk field whose spatial dissipation is not as diverse as is evident in Fig. 1 (for the time series given by $|u(t)|$ ). The principal reason for this is that the distribution in amplitudes of the trajectories given in Fig. 2 are within a common range and thus the distribution of gradients for any trajectory is the same, i.e. given Eq. (9), the derivative of $u(t)$ must be zero mean Gaussian distributed if $s(t)$ is so distributed. The principal difference between a random walk field based on Eq. (9) and that given in Fig. 1 is that in the former case, the field is ergodic [17] from one trajectory to the next.

For a system to be ergodic, any collection of random samples from a process must represent the average statistical properties of the entire process. In other words, regardless of what the individual samples are, a broad view of the collection of samples must represent the whole process. In the case of Fig. 1, it is clear that the statistical properties of the time series vary significantly from one country to another, specifically in regard to the standard deviation of the daily case time differences, i.e. the gradients of each time series. In this context, Eq. (9), and, the model it is predicated upon, fails to account for the differences in the infection rates that are observed. The solution to this issue is explored in the section that follows.

\section{Self-Affine Random Walk Fields}

The solution to Eq. (7) requires the distribution of the source term to be quantified and the memory function to be specified. In the latter case, let the memory function be given by 


$$
m(t)=\frac{1}{\Gamma(1-\alpha) t^{\alpha}}, \alpha \in(0,1)
$$

where $\Gamma$ is the Gamma function. The reason for adopting this particular function is that, as shall now be shown, the solution to Eq. (7) can be shown to yield a self-affine time series which is characteristic of many natural random processes including those presented in Fig. 1. In this context, the aim is to both simulate and quantify the data field in Fig. 1, the quantification being compounded in the parameter $\alpha$. To do this, we note a key result which is that

$$
\frac{1}{(i \omega)^{\alpha}} \leftrightarrow \frac{1}{\Gamma(\alpha) t^{1-\alpha}}
$$

where $\leftrightarrow$ denotes Fourier transformation. Thus,

$$
m(t) \leftrightarrow \frac{1}{(i \omega)^{1-\alpha}} \Rightarrow N(\omega)=(i \omega)^{1-\alpha}
$$

given Eq. (6).

\subsection{Solution for Eq. (7)}

In Fourier space, Eq. (7) is given by (using the Convolution Theorem)

$$
i \omega \tau U(\omega)=N(\omega) S(\omega)
$$

where $U(\omega)$ and $S(\omega)$ are the Fourier transforms of $u(t)$ and $s(t)$, respectively. Thus, we can write

$$
U(\omega)=\frac{1}{i \omega \tau}(i \omega)^{1-\alpha} S(\omega)
$$

or, using the convolution theorem again,

$$
u(t)=\frac{1}{\tau \Gamma(\alpha) t^{1-\alpha}} \otimes s(t)
$$

The solution for $u(t)$ is then expressed in terms of the Riemann-Liouville (fractional) integral which is an icon of the fractional calculus, Liouville having been one of the first to consider the possibility of fractional calculus in 1832. The integral may be considered to be the anti-derivative of a fractional differential and one of its principal properties is its scale invariance, given that for some scale length $\lambda>0$ (in this case, a scale in time) and using a change of variable, it can be shown that 


$$
u_{\lambda}(t)=\frac{1}{\tau \Gamma(\alpha) t^{1-\alpha}} \otimes s(\lambda t)=\frac{u(\lambda t)}{\lambda^{\alpha}}
$$

and we can therefore write

$$
u(\lambda t)=\lambda^{\alpha} u_{\lambda}(t)
$$

For this reason, the function $u(t)$ is a random scaling fractal and has a power spectral density function which scales with frequency as $1 /|\omega|^{2 \alpha}$ assuming that the power spectral density function for $s(t)$ is a constant, i.e. $s(t)$ is a 'white noise' source. This scaling law is a principal 'signature' for stochastic time series that exhibit random self-affine properties, the relationship between $\alpha$ and the fractal dimension $D \in(1,2)$ for such a time series being [18]

$$
\alpha=\frac{5}{2}-D
$$

\subsection{Solution for Eq. (8)}

Given Eq. (10), and, following the analysis given in Sect.4.1, Eq. (8) becomes

$$
u(t)=-\frac{1}{\tau \Gamma(\alpha) t^{1-\alpha}} \otimes u(t)+\frac{1}{\tau \Gamma(\alpha) t^{1-\alpha}} \otimes s(t)
$$

which has the transfer function

$$
T(\omega)=\frac{1}{1+\tau(i \omega)^{\alpha}}
$$

One approach to solving Eq. (12) is to apply iteration when

$$
u^{(k+1)}(t)=-\frac{1}{\tau \Gamma(\alpha) t^{1-\alpha}} \otimes u^{(k)}(t)+\frac{1}{\tau \Gamma(\alpha) t^{1-\alpha}} \otimes s(t), k=0,1,2, \ldots
$$

where

$$
u^{(1)}(t)=\frac{1}{\tau \Gamma(\alpha) t^{1-\alpha}} \otimes s(t)
$$

The first iteration is then equivalent to Eq. (11). In this case, a condition for the convergence of the solution must be investigated and obtained. Another approach to the problem is to consider the relationship between Eqs. (11) and (12) in terms of their respective transfer functions. This is the approach that is considered here, as shall now be addressed.

Since the transfer function for Eq. (11) is $1 / \tau(i \omega)^{\beta}, \beta \in(0,1)$, if we can relate $\alpha$ to $\beta$, then it becomes possible to compare the solution given by Eq. (11) and the 


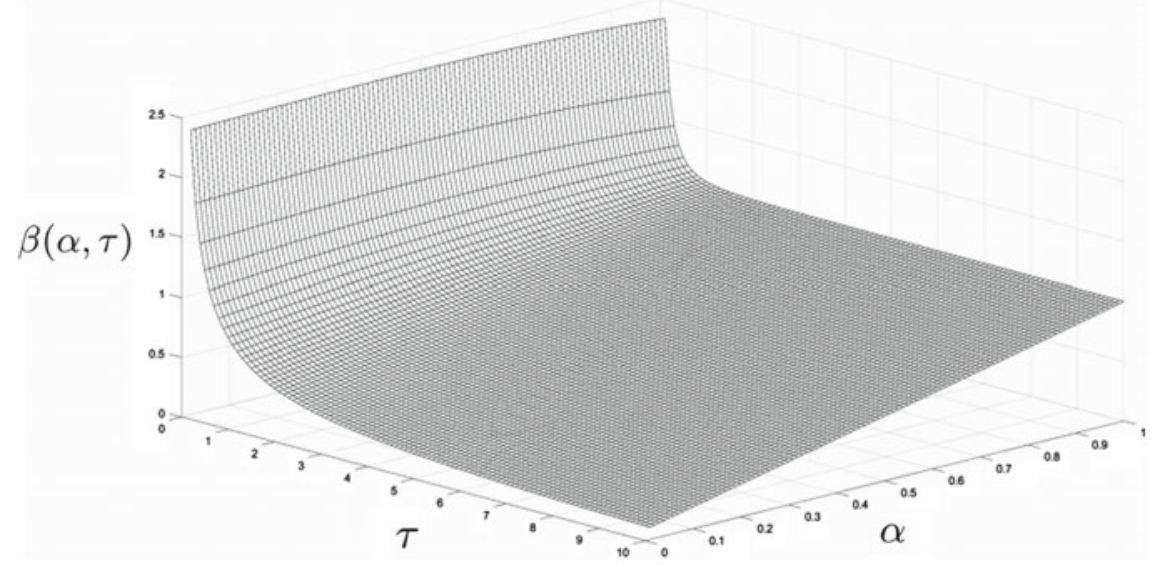

Fig. 3 Surface plot of $\beta(\alpha, \tau), \alpha \in(0,1), \tau \in(0,10]$ based on Eq. (14) illustrating that for $\tau>>$ 1 , there is a linear relationship between $\alpha$ and $\beta$.

solution we now require to Eq. (12). To do this, we equate the power spectra of the two transfer functions. This yields a relationship between $\alpha$ and $\beta$ for $\tau$ given by

$$
\tau^{2}|\omega|^{2 \beta}=1+\tau^{2}|\omega|^{2 \alpha}+2 \tau|\omega|^{\alpha} \cos (\alpha \pi / 2)
$$

so that upon setting $\omega=e$ we can write

$$
\beta=\frac{1}{2} \log \left[1+\tau^{2} \exp (2 \alpha)+2 \tau \exp (\alpha) \cos (\alpha \pi / 2)\right]-\log \tau
$$

Figure 3 shows a plot of $\beta$ for $\alpha \in(0,1)$ and $\tau \in(0,10]$ based on Eq. (14) and illustrates that as $\tau>1$ increases, there develops a linear relationship between $\alpha$ and $\beta$. In particular, for $\tau=10$, a linear fit between the two parameters yields $\beta=$ $0.9016 \alpha+0.09393$. Since Eqs. (11) and (12) are both scaled by $1 / \tau$, this result implies that for $\tau>>1$ the two equations are equivalent. Thus, the solution for $u(t)$ given by Eq. (11) is equivalent to the solution for $u(t)$ given by Eq. (12) with $\alpha \simeq 1.1 \beta-0.1$. Moreover, any estimate for $\alpha$ given $u(t)$ based on Eq. (11) through application of a regression analysis is simply related to an estimate for $\alpha$, given Eq. (12). For this reason, in the following section, we focus on the time series model compounded in Eq. (11).

\subsection{Random Walk Analysis}

Equation (11) can be written in the form 


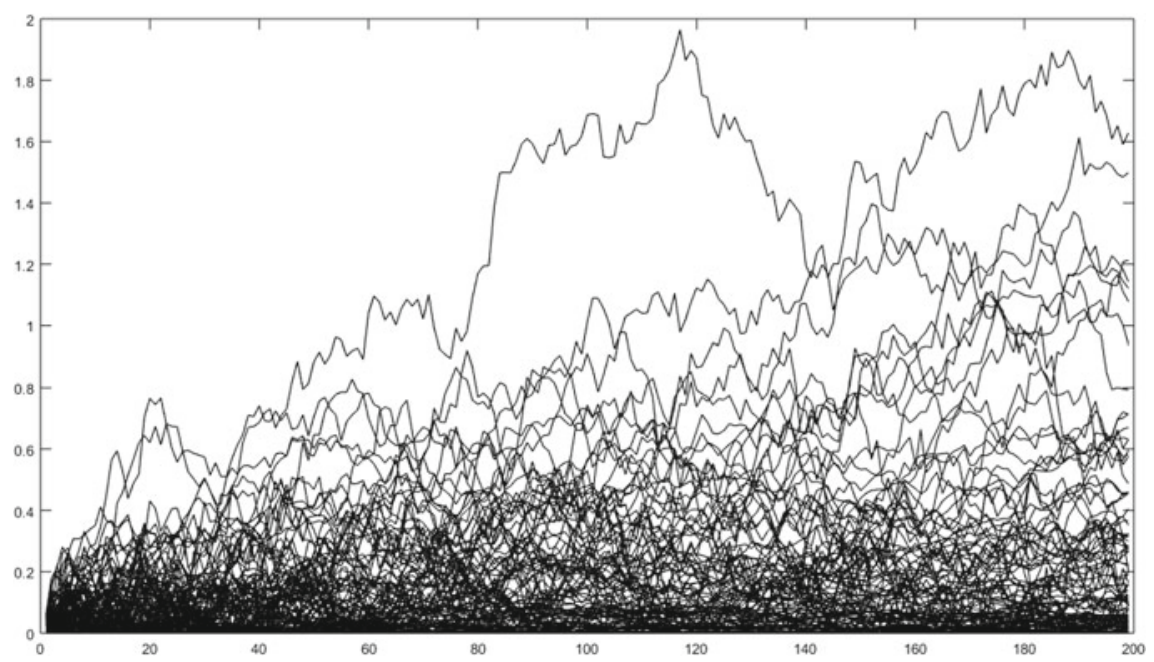

Fig. 4 Example simulation of a self-affine random walk field $|u(t)|$ for $\tau=10$ using Eq. (15) when $\alpha \in(0,1)$ is assumed to be a random Gaussian distributed variable

$$
\tau \frac{d}{d t} u(t)=\frac{d}{d t}\left(\frac{1}{\Gamma(\alpha) t^{1-\alpha}}\right) \otimes s(t)=\frac{1}{\Gamma(\alpha) t^{1-\alpha}} \otimes \frac{d}{d t} s(t)
$$

This provides an equation that is compatible with Eq. (9) so that, on comparing Eq. (9) with Eq. (15), it is apparent that if $\alpha$ is the same for all random walk trajectories the random walk field will be ergodic. However, if $\alpha$ varies randomly from one country to another, a non-ergodic random walk field will result of the type that is evident in Fig. 1. In this regard, the value of $\alpha$ determines the relative rate of growth of a trajectory and it is clear that as $\alpha \rightarrow 0$, any rate of growth becomes increasing suppressed because $\Gamma(\alpha) \rightarrow \infty$ as $\alpha \rightarrow 0$. This characteristic is reflected in Fig. 4 which shows an example random walk field $|u(t)|$ for $\tau=10$ based on Eq. (15) where $\alpha \in(0,1)$ is chosen from a zero mean Gaussian distributed source (by taking the absolute value of the output array and normalising the result) .

An essential difference between this model and that presented in Sect. 3 is that the non-ergodic characteristics of random walk field - specifically, the standard deviation of the gradients of $u(t)$ - are a measure of the random changes in the value of $\alpha \in(0,1)$ for each country. This is a consequence of the self-affine model developed through the application of the memory function given by Eq. (10).

\subsection{Example Results}

Based on the model for the memory function given by Eq. (10), $\alpha$ determines the memory of the system. The memory decays faster as $\alpha \rightarrow 1$ when one can intuitively 
expect the random walk field to have greater dispersion. In this sense, $\alpha$ can be interpreted as a measure of 'control' on the rate of infection which in turn, is related to issues such a lock-down and herd immunity. Thus, in the context of the selfaffine random walk model being considered, the infection will be suppressed when $\alpha \rightarrow 0$ for all countries (assuming travel continues form one country to another). Consequently, the evaluation of $\alpha$ on a country by country basis using available data is informative as given in Fig. 5 for 100 randomly selected countries. The basis for these results is the application of a least squares regression method to compute $\alpha$ given that (for a constant $C$ )

$$
\log |U(\omega)|^{2}=C-2 \alpha \log (\omega), \omega>0
$$

where $|U(\omega)|^{2}$ is the power spectrum of the data plotted in Fig. 1 for each country.

The results in Fig. 5 illustrate some important features that include the following:

(i) The values of $\alpha$ are not confined to the condition $\alpha \in(0,1)$;

(ii) there is a significant diversity in the values of $\alpha$ computed;

(iii) the distribution of values for $\alpha$ decays with higher values;

(iv) the mean value of $\alpha$ is 0.5746 .

The reasons for point (i) above are that the quantity of data currently available is not significant enough to provide accuracy on the computation of $\alpha$ through the regression method used (i.e. a least squares estimate of $\alpha$ ). Another issue is that for some countries, the model for $u(t)$ being considered may not conform to the data. Hence, the results shown in Fig. 5 should not be taken to be statistically significant. It is expected that the significance of such results will improve as further data becomes available. Nevertheless, in regard to point (ii) above, the results illustrate that there is, as would be expected, significant diversity in the random walk fields given in Fig. 1 for the Covid-19 pandemic, irrespective of directives from the World Health Organisation, for example.

By way of some specific examples, compared to Sweden, $\alpha$ is larger for both the USA and the UK, for example, that have introduced lock-down policies [19]. Sweden has endorsed a policy not to lock down the country in response to the global pandemic. Thus, on the basis of Eq. (11), and its interpretation with regard to the pandemic, Sweden is better served by the policies the Health authorities have introduced [20], as predicated on the self-affine random walk approach being considered in this work.

It should be noted that as $\alpha$ approaches 0 , the memory function given by Eq. (10) becomes constant in time, i.e. $m(t) \rightarrow 1$ as $\alpha \rightarrow 0$ when $\Gamma(1-\alpha)=1$. A memory function that is constant in time implies that the density field $u$ is time invariant. This is because, from Eq. (4),

$$
\tau m(t) \otimes \frac{\partial}{\partial t} u(\mathbf{r}, t)=\tau u(\mathbf{r}, t) \otimes \frac{d}{d t} m(t)=0, \text { if } m(t)=1 \forall t
$$

and we are then left with the time-independent equation 

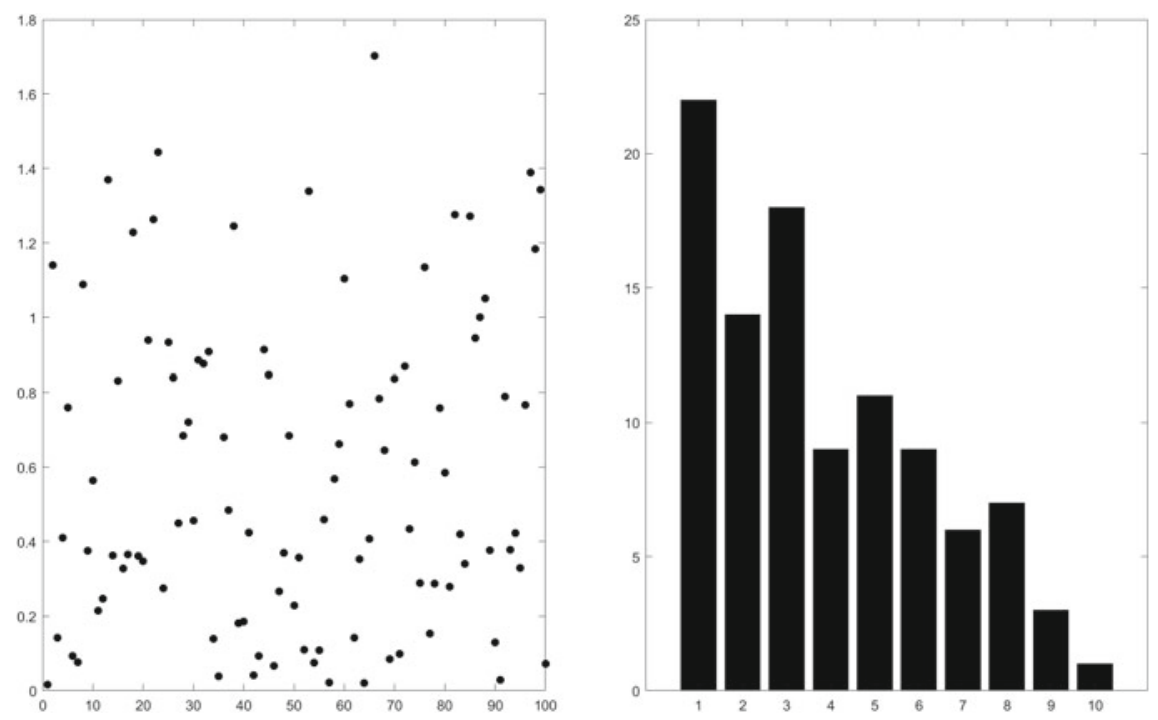

Fig. 5 Values of $\alpha$ for 100 randomly selected countries (left) and the associated 10-bin distribution (right)

$$
u(\mathbf{r}) \otimes p(\mathbf{r})+s(\mathbf{r})=u(\mathbf{r})
$$

From Eq. (11), it is clear that $u(t) \rightarrow 0 \forall t$ as $\alpha \rightarrow 0$ when the density field ceases to evolve in time, its spatial distribution being determined by the solution to Eq. (16). In this context, if $p(\mathbf{r})=\delta^{n}(\mathbf{r})$, then $s(\mathbf{r})=0$. On the other hand, for a Lévy distribution with approximated Characteristic Function (for Lévy Index $\gamma$ )

$$
P(\mathbf{k})=\exp \left(-|k|^{\gamma}\right) \sim 1-|k|^{\gamma}, \gamma \in(0,2)
$$

we can write Eq. (16) in terms of the fractional Poisson equation

$$
\nabla^{\gamma} u(\mathbf{r})=s(\mathbf{r})
$$

where $\nabla^{\gamma} u(\mathbf{r}) \leftrightarrow-|\mathbf{k}|^{\gamma} U(\mathbf{k})$. The relationship between $\gamma$ and the fractal dimension is given by [18]

$$
\gamma=\frac{3}{2} n+1-D, \mathbf{r} \in \mathbb{R}^{n}
$$

Thus, for example, when $n=2$, Eq. (17) is the equation for a Mandelbrot surface [18]. 


\section{The Bio-Dynamics Hypothesis}

The Bio-dynamics Hypothesis is concerned with the connectivity between the dynamical behaviour of bio-organisms in regard to their replication and growth and the geometrical structures of the physical forms that result. It states that if the growth of a bio-organism is self-affine, then the geometry of the organism will also be selfaffine.

In this section, we consider a similar model to that discussed in Sect. 4 but with a focus on developing a parametric representation whose aim is to reflect the selfaffine structures of proteins. The reason for this is that understanding and interpreting the structure (geometric configuration) of the spike protein on a coronavirus is the key to developing a vaccine. This is because it is through the spike protein that the virus attaches, fuses and gains entry to cells. Therefore, analysis of the spike protein 'architecture' coupled with its mechanics is vital in revealing information that can prompt the discovery of countermeasures against the virus.

In this context, the basic principle for developing a vaccine is well known. If a protein can be found that has the same structure as the spike protein and is introduced into the body prior to infection, then anti-bodies will be generated by the body that will destroy the virus by suppressing its ability to replicate through elimination of the spike protein. On this basis, what is required is a search and/or fabrication of proteins whose self-affine structure is the same as the spike protein.

\subsection{Self-affine Structures of a Virus}

It is well known that there is a correlation between the effect (in particular, its fatalness) of a virus and its fractal geometry. This refers primarily, but not exclusively, to the surface roughness of a virus and its metabolic rate. In turn, the surface roughness is related to the structural complexity of the spike proteins and their density on the surface of the virus. For a single near spherical cell, its metabolic rate, $M_{R}$, scales as $M_{R} \sim r^{2}$ where $r$ is the radius of the cell but for a virus, its metabolic rate scales as $M_{R} \sim r^{D}$ where $D \in(2,3)$ is the fractal dimension of the surface and $r$ is the characteristics radius of the virus [21].

Figure 6 shows a comparison of the simulated structures for the spike proteins of the Covid-19 virus, the influenza virus and the Human Immunodeficiency Virus (HIV). In each case, the spike protein is not one continuous feature but is composed of specific protein strands which are colour coded. While the Covid-19 spike protein displays structural similarities to the spike proteins of influenza virus and HIV, it is, by comparison, the largest class I fusion protein known to date. Given that Covid-19 is new and there is no general immunity to it, this is why it is so relatively dangerous.

Use of Eq. (17) to model such structures is not relevant. This is due to the following: 


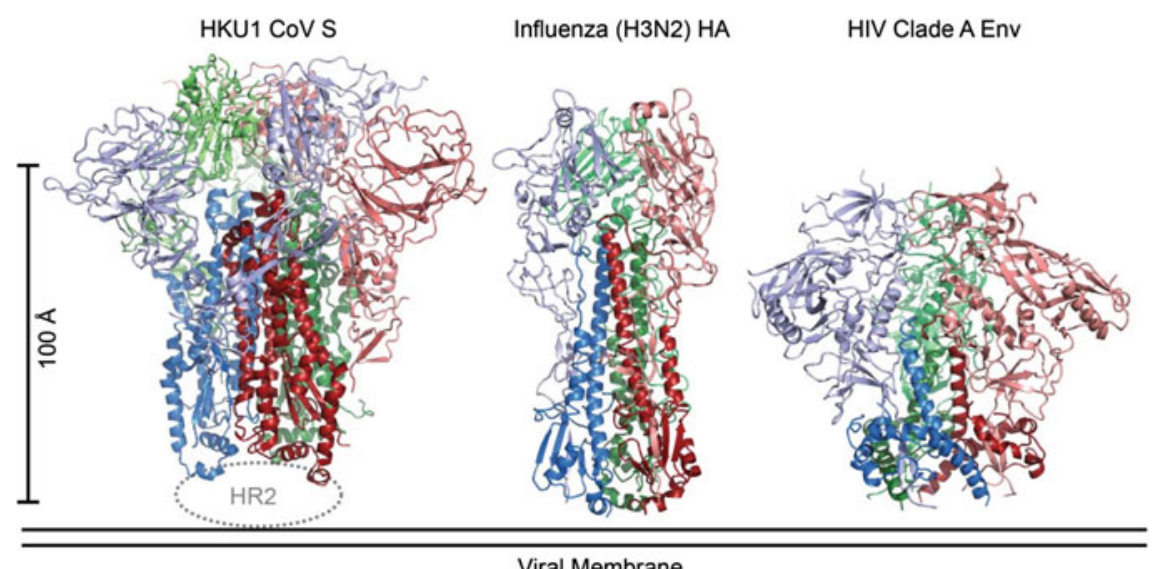

Viral Membrane

Fig. 6 Comparison of the simulated structural complexity of the spike protein's for coronavirus (CoV S), the influenza virus and HIV [22]

- the equation assumes that the evolution process is time-independent;

- it is not compatible with modelling a self-affine field that is based on long continuous strands of which proteins are an important example.

Instead, we consider a parametric self-affine model which is discussed in the following section.

\subsection{A Parametric Self-affine Model}

For $p(\mathbf{r})=\delta^{n}(\mathbf{r})$, Eq. (5) reduces to

$$
\tau \frac{\partial}{\partial t} u(\mathbf{r}, t)=n(t) \otimes s(\mathbf{r}, t)
$$

Consider a solution to this equation based on an additive separation of variables when

$$
u(\mathbf{r}, t)=u_{x}(t)+u_{y}(t)+u_{z}(t) \text { and } s(\mathbf{r}, t)=s_{x}(t)+s_{y}(t)+s_{z}(t) .
$$

The source function is taken to model a system characterised by a set of additive spatial sources which may have independent stochastic properties. We are then interested in the evolution of a density field in a three-dimensional space that is taken to be described by the parametric curve $\left[u_{x}(t), u_{y}(t), u_{z}(t)\right]$.

Following the solution method discussed in Sect. 4 for the memory function given by Eq. (10), we can write the solution for each component of the parametric curve as 

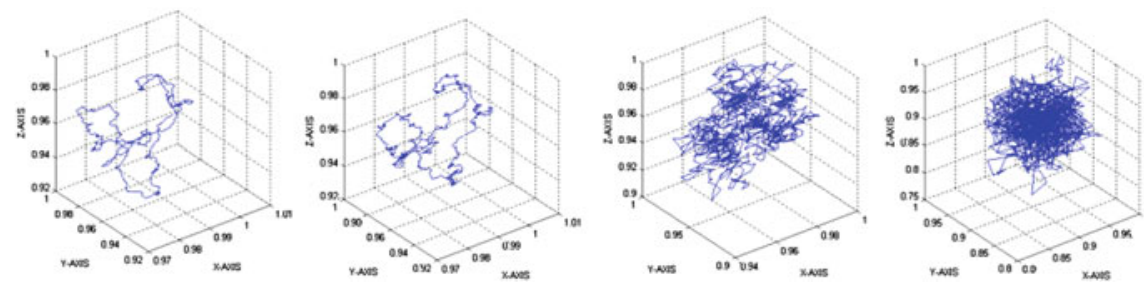

Fig. 7 Evolution of a three-dimensional parametric curve for (from left to right consecutively) $\alpha=0.8, \alpha=0.6, \alpha=0.4$ and $\alpha=0.2$ obtained using Eq. (18)

$$
\left[\begin{array}{l}
u_{x}(t) \\
u_{y}(t) \\
u_{z}(t)
\end{array}\right]=\frac{1}{\tau \Gamma(\alpha) t^{1-\alpha}} \otimes\left[\begin{array}{l}
s_{x}(t) \\
s_{y}(t) \\
s_{z}(t)
\end{array}\right]
$$

The Fourier space representation of this result is

$$
\left[\begin{array}{l}
U_{x}(\omega) \\
U_{y}(\omega) \\
U_{z}(\omega)
\end{array}\right]=\frac{1}{\tau(i \omega)^{\alpha}}\left[\begin{array}{l}
S_{x}(\omega) \\
S_{y}(\omega) \\
S_{z}(\omega)
\end{array}\right], \omega>0
$$

where the upper case functions of the angular frequency $\omega$ denote the Fourier transforms of the corresponding lower case function of time. It is then clear that the parametric curve is composed of elements that are characterised by the same value of $\alpha$. Although the source functions are uncorrelated, their power spectral density functions are taken to be the same. The structure of the parametric curve is then determined by the value of $\alpha$ which, in turn, is a measure of the influence of the memory function in time. This is illustrated in Fig. 7 which shows parametric curves for various values of $\alpha \in(0,1)$ illustrating that the complexity of the curve increases as the value of $\alpha$ decreases.

\subsection{Discussion}

If we can model the Covid-19 spike protein as a parametric curve with a known value $\alpha$ based on Eq. (18), then it may be possible to use this value in the search for other benign proteins that have a similar $\alpha$ value. Such proteins would then at least possess the same structural complexity which is an important factor in the development of any vaccine. One way to determine the value of $\alpha$ from protein models of the type given in Fig. 6 is to extract data associated with the functions $u_{x}(t), u_{y}(t)$ and $u_{z}(t)$ (essentially the coordinate values of the structure obtained by moving along the protein strand), evaluate $\alpha$ in each case and compute the mean value. 


\section{Summary, Conclusions and Future Research}

The material presented in this work has been developed to provide readers with an overview of the ways in which Eq. (2) can be used as a frame work for investigating epidemic and pandemic time series (and viral structures). In this context, a summary of the material presented is now given followed by some conclusions and directions for future research.

\subsection{Summary}

The evolution equation for a stochastic source given by Eq. (2) is a fundamental field equation of statistical mechanics. It is applicable in all topological dimensions and for any system composed of random interactions (elastic scattering). In the application of Eq. (2) to the evolution of an infectious disease, these interactions are taken to be the transmission of a pathogen from one person to another. In this regard, the focus of the work has been to develop time series models for the evolution of Covid-19 informed by the data that is currently available. For this purpose, and, using Eq. (4), a memory function has been considered-Eq. (10) — that yields a self-affine model for the time series. The reason for doing this is because it is known that biological and bio-medical time series are self-affine which is entirely compatible with the fractal geometry of nature [23]. Not surprisingly, therefore, this also appears to be the case for Covid-19 pandemic time series data.

Two time-only dependent equations have been derived, namely Eq. (7) and Eq. (8). The former equation is based on assuming the PDF in Eq. (2) is a delta function and corresponds to the case when $\tau<<1$. The latter equation is based on an asymptotic solution and is applicable for any PDF $p(\mathbf{r})$ which approaches zero as $r \rightarrow \infty$ and valid for all values of $\tau$. However, through an analysis of the transfer functions for both equations as given in Sect. 4.2, it has been shown that the two equations have an equivalence in terms of the relationship between $\alpha$ for each equation as given by Eq. (14). This avoids having to resort to an iterative approach for solving Eq. (8). Moreover, it provides a method of determining the parameter $\alpha$ for Eq. (8) based on applying a recursion analysis using Eq. (7), i.e. given $u(t)$, compute $\alpha$.

\subsection{Conclusions}

The application of stochastic models avoids the indeterminacy associated with implementing a deterministic model with many coefficients. On a global perspective, this approach assumes that there are no intrinsic correlations between the dynamics of the pandemic and its intervention and control. This is due to the multifaceted differences that are being introduced by different governments through different policies at dif- 
ferent times in order to attempt to reduce the severity of the disease using 'solutions' that are not, as yet, fully proven (i.e. statistically significant), e.g. [25].

The models and results presented in this work are developments based exclusively on the evolution equation-Eq. (2). In this context, the time series model compounded in Eq. (11) is critically dependent on the memory function parameter $\alpha$. From the example time series data given in Fig. 1, it is clear that the stochastic characteristics are non-ergodic (i.e. they changes significantly from one country to another) and that $\alpha$ changes from one country to another. Thus, the growth of the pandemic in each country is memory dependent. The distribution of $\alpha$ presented in Fig. 5 is informative but not yet statistically significant due to the lack of data that is currently available. Consequently, the results given in Fig. 5 need to be continually re-evaluated as the Covid-19 pandemic evolves.

The value of $\alpha$ provides a measure on the dissipation of the pandemic. It has a synergy with the reproduction number $R$ associated with deterministic models which is a way of rating the ability for an infection to spread-the number of people (on average) that one infected person will pass the virus on to. To extinguish an infectious disease, we require that $R<1$. To extinguish an infectious disease based on a selfaffine evolutionary model based on Eq. (8), we require that $\alpha \rightarrow 0$. This is because $u(t)$ approaches zero due to the scaling of Equations (11) and (12) by $1 / \Gamma(\alpha)$, i.e. $\Gamma(\alpha) \rightarrow \infty$ as $\alpha \rightarrow 0$. However, it is arguable that this is not physically possible, because it implies that the memory function is constant in time which in turn implies that the system is time-independent and that there is no time evolution of the density field.

On the basis of the evolution equation and the analysis used to derive a time-onlydependent representation for the density field $u(t)$, Equations (11) and (12) provide basic stochastic time series models which have an intrinsic relationship. If $|u(t)|$ is taken to be a model for the infections over a uniform period of time (e.g. each day), then the amplitude of this function is reduced as $\alpha \rightarrow 0$ and as $\tau \rightarrow \infty$. In terms of the dynamics of a global pandemic, this result implies that the longer the memory associated with a population maintaining a high value of $\tau$ is (giving a low infection rate), the greater the rate at which $|u(t)|$ reduces to zero, thereby extinguishing the pandemic.

In the same way that $\alpha$ is a gauge on viral infection rates, in the context of the Fig. 6 and the Bio-dynamics Hypothesis, it also relates to the self-affine structures that are prevalent in the spike protein. In both cases, the value of $\alpha$ determines the rate of infection and the structure of the protein that is causing the disease. The interesting question is whether there is a correlation between the two, i.e. the mean value of $\alpha$ that is characteristic of the infection rate in a global context and the value or $\alpha$ that is characteristic of the structural complexity of the spike protein.

The underlying principle is that the Covid-19 virus will fractionally diffuse in the early stages of the pandemic exhibiting self-affine characteristics, and, as time increases, the dynamics will become increasingly characterised by classical diffusion. This is a consequence of the Central Limit Theorem when $\gamma \rightarrow 2$ as $t \rightarrow \infty$. Further, as time increases, the value of $\alpha$ can be expected to approach zero for all countries thereby giving the appearance of the pandemic becoming extinct, the final density 
field then being given by Eq. (17) for $\gamma=2$, i.e. the time-independent diffusion equation for a source.

In the context of this conceptual interpretation, the effect of attempting to control the infection rates will merely delay the transition from fractional to classical diffusion. Compared to the 1918 'Spanish flu' pandemic, for example, which involved the H1N1 virus, taking a few years to be extinguished at the cost of many tens of millions lives [24], the current pandemic is likely to continue for a longer period of time but at the same final cost. This is not due to a difference in the dynamical behaviour of Covid-19 now compared to H1N1 in 1918, but the considerable difference in the moral imperatives of today compared to 100 years ago. In this regard, the failure of deterministic pandemic models is in part due to 'interference' of central governments and their time varying policies which alter the dynamics of the infection rate, one that is randomly inhomogeneous on a worldwide basis. Thus, a stochastic approach to modelling the pandemic is, in part, necessitated by the moral imperatives that modern governments are expect to adopt which injects randomness into the evolution of the disease when viewed on a global perspective [25]. On the basis of the models developed and the data analysed, this randomness appears to be self-affine. In this context, the models presented reflect the inconsistencies of implementing a lock-down and substantiates, on a theoretical level at least, the importance of evidence-based medicine rather than highly sensitive deterministic modelling based on assumptions and many unknowns [26] as briefly discussed in the introduction.

By way of an analogy, consider an ink drop which is introduced to the surface of some water that is contained in a vessel where it is assumed that the ink is the same density as the water and that the water is at a constant temperature and is homogeneous throughout the container. The ink will flow into the water producing complex patterns while spreading away from the point on the surface at which it has been introduced. These complex patterns represent the combined effects of each ink molecule undertaking a random walk. As time increases, the ink will diffuse into the water and eventually become equally distributed throughout, a process that is irreversible. A lock down is then analogous to draining the container by introducing a channel at the bottom of the vessel, for example, at the time the ink is first introduced in order to try and eradicate its presence. The effect of this is to drain some of the ink but at the expense of a falling water level. The difference between the initial and final water levels is then analogous to the difference between the economic prosperity of a country before and after lock down, especially in regard to the younger and more healthy component of the population who are inherently less vulnerable to the effects of infection. The economic effects of this may be destined to be significant [27]. In this context, the objective to suppress the pandemic through intermittent lock-downs, while waiting for an effective vaccine to be developed is, while laudable, not feasible and may lead to significant long-term damage, especially to those who are at the forefront of wealth creation in a society. 


\subsection{Future Research}

Within the context of the material presented, example suggestions for future work include the following:

- Simulation of Covid-19 random walk fields using Eq. (12) for distributions in the values of the infection rate $\tau$;

- evaluation of distributions for $\alpha$ using different data associated with the Covid-19 pandemic;

- evaluation of the time evolution of $\alpha$ as more data becomes available;

- evaluation of $\alpha$ using more advanced regression methods such as singular value decomposition which typically requires extensive data sets;

- simulation of spike proteins based on Eq. (18) when the value of $\alpha$ varies for each component of the parametric curve;

- computation of $\alpha$ for different continuous strands of proteins from which the spike protein is composed based on Eq. (18);

- analysis of solutions to Eq. (5) using time series data predicated on spatial locations, i.e. the geographical location of a country relative to a common origin such as Wuhan in China, for example (where the Covid-19 virus is considered to have emerged) and the location of isolated pockets of infections with each country as and when such data becomes available.

\section{References}

1. Han, et al.: Novel Coronavirus Pneumonia (COVID-19) progression course in 17 discharged patients: comparison of clinical and thin-section CT features during recovery. Clin. Infect. Dis. 71(15), 723-731 (2020). https://doi.org/10.1093/cid/ciaa271

2. Bacaër, N.: McKendrick and Kermack on Epidemic Modelling (1926-1927). In: A Short History of Mathematical Population Dynamics, pp. 89-96. Springer, London (2011). https://doi.org/ 10.1007/978-0-85729-115-8_16

3. Jones, D., Helmreich, S.: A history of herd immunity, perspectives, the art of medicine. The Lancet 396 (2020). https://www.thelancet.com/journals/lancet/article/ PIIS0140-67362031924-3/fulltext

4. Siettos, C.I., Russo, L.: Mathematical Modelling of infectious Disease Dynamics, vol. 4(4), pp. 295-306. Taylor \& Francis, Virulence (2013). https://www.tandfonline.com/doi/pdf/10. 4161/viru.24041

5. Cakan, S.: Dynamic analysis of a mathematical model with health care capacity for COVID-19 pandemic. Chaos, Solit. Fractals 139, 110 033. Elsevier (2020)

6. Dayaratna, K.: Failures of an Influential COVID-19 Model Used to Justify Lockdowns. The Heritage Foundation. https://www.heritage.org/public-health/commentary/ failures-influential-covid-19-model-used-justify-lockdowns. Accessed 21 Sept 2020

7. Crank, J.: The Mathematics of Diffusion. Clarendon Press, Oxford (1956)

8. Wyss, W.: The fractional diffusion equation. J . Math. Phys. 27, 2782 (1986). https://doi.org/ $10.1063 / 1.527251$

9. Blackledge, J.M., Barry, D.: Morphological analysis from images of hyphal growth using a fractional dynamic model. In: Carr, H., Grimstead, I. (eds) EG UK Theory and Practice of Computer Graphics (Warwick University), 17-24 (2011) https://doi.org/10.21427/3ssf-3335 
10. Melin, P., Monica, J.C., Sanchez, D., Castillo, O.: Analysis of spatial spread relationships of Coronavirus (COVID-19) pandemic in the world using self organizing maps. Chaos, Solit. Fractals, Elsevier 138, 109917-109918 (2020). https://doi.org/10.1016/j.chaos.2020.109917

11. Einstein, A.: On the motion of small particles suspended in liquids at rest required by the molecular-kinetic theory of heat. Annalen der Physik 17, 549-560 (2016)

12. Samorodnitsky, G., Taqqu, M.S.: 1994. Stochastic Models with Infinite Variance, CRC Press, Stable Non-Gaussian Random Processes (1994)9780412051715

13. Kolmogorov, A.N.: On analytic methods in probability theory. In: Shiryaev, A. N. (ed) Selected Works of A. N. Kolmogorov, Volume II: Probability Theory and Mathematical Statistics1992, Kluwer, Dordrecht, 61-108 (1992). Based on the Original: Uber die Analytischen Methoden in der Wahrscheinlichkeitsrechnung, Math. Ann., 104, 415-458 (1931)

14. Feller, W.: On boundaries and lateral conditions for the Kolmogorov differential equations. Ann. Math., Second Series 65(3), 527-570 (1957)

15. Evans, G., Blackledge, J.M., Yardley, P.: Analytic Solutions to Partial Differential Equations, Springer Undergraduate Mathematics Series, Springer-Verlag. London (1999). https://doi.org/ 10.1007/978-1-4471-0379-0

16. Our World in Data, Coronavirus (COVID-19) Cases (2020). https://ourworldindata. org/coronavirus-data-explorer?Scale=log\&zoomToSelection=true \&casesMetric=true \& interval=smoothed\&aligned $=$ true\&smoothing $=7 \&$ country. Accessed 21 Sept 2020

17. Papoulis, A.: Probability, pp. 427-442. Random Variables and Stochastic Processes, McGrawHill, New York (1991)0-07-048477-5

18. Turner, M.J., Blackledge, J.M., Andrews: Fractal Geometry in Digital Imaging, Academic Press, P. R. (1998)-10: 0127039708

19. Giesecke, J.: Why Lockdowns are the Wrong Policy, LockdownTV, Unherd.com/live ,17 April, 2020. https://www.youtube.com/watch?v=bfN2JWifLCY\&feature=youtu.be. Accessed 20 Sept 2020

20. Neil A.: Interviews Anders Tegnell - A Second Wave and What Sweden Got Right I SpectatorTV, 18 Sep 2020. https://www.youtube.com/watch?v=6C99MtK4ogM. Accessed 21 Sept 2020

21. He, J.H.: Fatalness of virus depends upon its cell fractal geometry. Chaos, Solit. Fractals 38, 1390-1393 (2008)

22. Scripps Research Institute, TSRI Scientists find Clues to Neutralizing Coronaviruses such as MERS, Public Release, 2 March, 2016. https://www.eurekalert.org/pub_releases/2016-03/sritsf022916.php. Last accessed 12 August, 2020

23. Mandelbrot, B.B.: The Fractal Geometry of Nature. W. H, Freeman and Co (1982)0-71671186-9

24. Center's for Disease Control and Prevention, 1918 Pandemic (H1N1 virus) (2020). https://www. cdc.gov/flu/pandemic-resources/1918-pandemic-h1n1.html. Last accessed 12 August 2020

25. Gandhi, M., Rutherford, G.: Facial Masking for Covid-19 - Potential for 'Variolation' as we Await a Vaccine, Perspective, The New England Journal of Medicine, Massachusetts Medical Society, 1-3, September 8, (2020). https://doi.org/10.1056/NEJMp2026913

26. Kirkham, P. Yeadon, M., Thomas, B.: How Likely is a Second Waves, Lockdown Sceptics, 8 September (2020). https://lockdownsceptics.org/addressing-the-cv19-second-wave. Accessed 20 Sept 2020

27. Varoufakis, Y.: Something Remarkable just Happened this August: How the Pandemic has Sped up the Passage to Post-Capitalism, 25 August, 2020 https://diem25. $\mathrm{org} /$ something-remarkable-just-happened-this-august-how-the-pandemic-has-sped-thepassage-postcapitalism. Accessed 20 Sept 2020 